Bangladesh J. Bot. 49(4): 1085-1093, 2020 (December)

\title{
ANTIMICROBIAL ACTIVITIES OF CRUDE SOLVENT EXTRACT AND SYNTHESIZED SILVER NANOPARTICLES OF OPUNTIA FICUS-INDICA L. CLADODES
}

\author{
IF Bahis, Mahmoud Moustafa*1 and Hussein Al-W adi \\ Department of Biology, College of Science, King Khalid University, \\ 9004, Abha, Kingdom of Saudi Arabia
}

Keywords: Antimicrobial activity, Opuntia ficus-indica, Silver nanoparticles

\begin{abstract}
Opuntia ficus-indica cladodes was examined for their antimicrobial activities by using crude extract and synthesized silver nanoparticles against human pathogenic microbial strains, namely Staphylococcus aureus, Klebsiella pneumoniae, Klebsiella oxytoca, Proteus mirabilis and Candida albicans. All extracts demonstrated moderate activity against the tested microbes in a range between 0.53 and $2.33 \mathrm{~cm}$. The maximum inhibition activity was found to be against $K$. pneumoniae while the lowest against $S$. aureus. Synthesized silver nanoparticles (Ag-NPs) showed inhibition activities between 1.82 and $2.03 \mathrm{~cm}$. The maximum activity was recorded against $K$. oxytoca, while the lowest activities against $P$. mirabilis. In context of antimicrobial activity, there was no significant difference either solvent crude extract or synthesized silver nanoparticles against tested microbes. Therefore, crude extracts of $O$. ficus-indica cladodes or Ag-NPs could be used as an alternative natural drug.
\end{abstract}

\section{Introduction}

Nowadays the multidrug-resistant bacteria becoming a global risk to public health whereas many workers in recent years have focused on how to develop new antimicrobial agents from natural sources for combating such microbial strains (Martin et al. 2015). Finding cheaper, having a little side effect than the synthetic chemical alternative agents from plant sources is also other main goal for the researchers (Abouhosseini et al. 2012, Dastagir et al. 2012). Opuntia sp. had been extensively studied for their antiviral, antitumor, cytotoxic, antimicrobial, and for their pesticidal activities (Galati et al. 2007, El-Feghali et al. 2018). Chemical analysis showed that the cladodes of $O$. ficus-indica consist of polyphenols and vitamins that make it medically significant at the level of antioxidant, antimicrobial, and hypoglycemic and those in turn found to be effective against inflammation, heart problems, vascular disorders, and cancer (Trombetta et al. 2006). Previous study showed that the solvents extracted from $O$. ficus-indica cladodes and flowers had high-to-moderate antimicrobial activities against some bacterial and fungal pathogenic species (Ennouri et al. 2014, El-Feghali et al. 2018). O. ficus-indica was recorded in Central America (Mexico) and later, the species was introduced to many areas such as savanna regions inhabiting South Africa, Asia, and Australia. In other parts, it was introduced as an ornamental plant, for camel feeding, and for its delicious fruits as a food for humans. In alternative medicine, the fruits and stems of $O$. ficus-indica provide sources to treat digestive problems such as diabetes and burns (Lee et al. 2003).

Opuntia spp. extracts have complex compounds and pure secondary metabolites (Prabhu and Poulose 2012), hence this property should be exploited to reduce metal ions such as gold and silver into nanoparticles, an important phenomenon termed green biosynthesis. Applications of

*Author for correspondence: <mfmostfa@kku.edu.sa>. ${ }^{1}$ Department of Botany \& Microbiology, Faculty of Science, South Valley University, Qena, Egypt. 
nanoparticles have been succeeded in the medical field study as to aid the diagnosis of cancer as well as developing alternative antimicrobial drugs instead of industrial antibiotics as the resistant microbial strains being identified (Ahmed et al. 2016).

The plant distributed widely on Saudi Arabia especially in abandoned area and around the cultivated regions. Details about antimicrobial activities of the cladodes of $O$. ficus-indica invasive plant had been reported very little. Therefore, this attempt aimed at evaluating the antimicrobial activities of $O$. ficus-indica cladodes from either their solvent crude extracts or Ag-NPs extracts could be first report in the pharmaceutical area.

\section{Materials and Methods}

Opuntia ficus-indica L. fresh samples were collected in October 2017 from Raydah Reserve Mountains, Kingdom of Saudi Arabia. Some plant parts were kept in plastic bags and preserved in the Refrigerator at $4^{\circ} \mathrm{C}$ for further use. Three hundred grams of $O$. ficus-indica fresh cladodes were dried for three months under shade in $23^{\circ} \mathrm{C}$ and then ground to a fine powder by using a mortar and pestle for dry extraction. Fifty grams were crushed directly using an electric grinder to a fine solution for fresh extractions, then filtrated through using Whatman filter paper No. 1..

Five grams of powder from each plant material and mucilage solution were weighed in a test tube. Fifteen $\mathrm{ml}$ of the solvent including petroleum ether, 1-butanol, methanol, ethanol, and chloroform were added to each sample. All samples were placed in a rotary shaker at $150 \mathrm{rpm}$ at $37^{\circ} \mathrm{C}$ for $48 \mathrm{hrs}$ to allow complete extraction of chemical compounds. All solvents in each tube were evaporated using an oven at $59^{\circ} \mathrm{C}$ for one week. Weighted dry extracts were dissolved in 15 $\mathrm{ml}$ of dimethyl sulfoxide (DMSO) and $5 \mathrm{ml}$ for fresh samples and incubated in a rotary shaker at $150 \mathrm{rpm}$ at $25^{\circ} \mathrm{C}$ to ensure complete dissolving of crude plant extracts (Salvat et al. 2004).

The test microbial strains were obtained from the Microbiology Department, Faculty of Medicine, King Khalid University, Kingdom of Saudi Arabia. The strains included one Grampositive (S. aureus), three Gram-negative ( $P$. mirabilis, $K$. oxytoca and $K$. pneumoniae) bacteria, and one pathogenic fungus (C. albicans). All pathogenic strains were first sub-cultured in nutrient broth (NB) and incubated aerobically at $27^{\circ} \mathrm{C}$ for $24 \mathrm{hrs}$ for all bacterial isolates and for $48 \mathrm{hrs}$ for C. albicans.

Fifty various extracts in triplicate were tested for their antimicrobial activities against $S$. aureus, $P$. mirabilis, $K$. oxytoca, $K$. pneumoniae and $C$. albicans. Twenty $\mathrm{ml}$ from a previously made sterilized nutrient agar (NA) medium was transferred into sterile Petri plates and left for one hour at room temperature to allow for solidification. A total of three wells were made in each nutrient agar plate using a sterile cork-borer (6 mm in diameter). Each microbial strain of $0.1 \mathrm{ml}$ was spread over the solid agar using a sterilized loop. One hundred $\mu \mathrm{l}$ of the prepared plant extract was transferred into the wells using a sterilized pipette. The plates were then incubated at $37^{\circ} \mathrm{C}$ for $24 \mathrm{hrs}$. The diameters of the inhibition zone in centimeters were measured using a transparent ruler. Each experiment was repeated three times and the mean values were calculated (Moustafa et al. 2013).

Five grams of the cladodes from $O$. ficus-indica was transferred into $50 \mathrm{ml}$ of deionized water (DIW). All samples were placed into a rotary shaker at $150 \mathrm{rpm}$ at $25^{\circ} \mathrm{C}$ for three days to allow complete extraction. Extracts were filtered using Whatman filter paper No. 1 in a conical flask. The conical flask containing $25 \mathrm{ml}$ of $0.167 \mathrm{mM} \mathrm{AgNO}_{3}$ was heated at $70^{\circ} \mathrm{C}$ and the plant extracts were added drop by drop until the colloidal solutions turned yellow for the first ten minutes and turned dark brown after one hour and then kept at $4^{\circ} \mathrm{C}$ for further use. The absorbances of colloidal solutions were measured with a spectrometer (Beckman - Model No. DU-50, Fullerton, CA, USA). One $\mathrm{ml}$ of the suspension was collected to evaluate the completion of $\mathrm{Ag}^{+}$in aqueous 
solution, then diluted with an appropriate amount of deionized water and subsequently observed in the UV-visible spectra, between wavelengths of 400 and $700 \mathrm{~nm}$. Images of Ag-NPs were obtained using a scanning electron microscope (SEM; Hitachi S4800) equipped with an energydispersive X-ray spectroscope.

Antimicrobial activities of solutions containing nanoparticles synthesized from cladode part of $O$. ficus-indica were performed against various microbial strains. Twenty $\mathrm{ml}$ of the sterilized nutrient agar (NA) medium was transferred into sterile Petri plates and five wells were made. $0.1 \mathrm{ml}$ inocula were spread over the solid agar and then $100 \mu \mathrm{l}$ of Ag-NPs was filled in each well. Each extract was repeated three times and mean values were recorded. DMSO was used as a negative control while cefoxitin $(30 \mu \mathrm{g}))$ dissolved in $15 \mathrm{ml}$ DMSO was used as positive control. The generated data were analyzed using Graph Pad Prism 6 Demo (version 7.04).

\section{Results and Discussion}

This study achieved the synthesis of silver ( $\mathrm{Ag}$ ) nanoparticles from silver nitrate using the cladode extract powder of Opuntia ficus-indica. Addition of $O$. ficus-indica cladode extract to $0.167 \mathrm{mM} \mathrm{AgNO}{ }_{3}$ aqueous solution led to the appearance of a dark brown color in the solution after approximately $1 \mathrm{hr}$ of reaction, indicating the formation and stabilization of silver nanoparticles (Fig. 1). The silver nanoparticles appeared to be a reddish-brown color in water. This color may be due to excitation of surface plasmon vibrations in the silver nanoparticles, as mentioned in other study (Song and Kim 2009). The UV-vis spectra showed the maximum absorbance in range 420-470nm (Fig. 2). Moreover, a SEM/EDX analysis confirmed the elements present in the solution at the rate of $\mathrm{Ag}(74.33 \%), \mathrm{C}(8.09 \%)$ and $\mathrm{Cl}(11.95 \%)$ (Fig. 3). In addition, $O$. ficus-indica cladode extract consists of many phytochemicals that play an important role in reducing ions to form nanoparticle sizes. The carboxylic group significantly affected the surface of the nanoparticles, and controlled the stabilization process through electrostatic forces (Prabhu and Poulose 2012). The antimicrobial activity of extracts of $O$. ficus-indica cladode was tested against four pathogenic bacteria, and one pathogenic fungus. The study revealed variations in antimicrobial activities by applying various solvent plants extracted (dry and fresh extracts (Figs 4 and 5). DMSO, which was used as a negative control, demonstrated no inhibiting effects. The positive controls showed inhibition diameters ranging from $2.00 \pm 0.02-2.5 \pm 60.04 \mathrm{~cm}$ (Cefoxitin dissolved in DMSO). The findings of this study showed that solvent extracts of fresh cladode of $O$. ficus- indica including those from 1-butanol, methanol, and chloroform had significant antimicrobial properties (Fig. 5). On the other hand, the maximum inhibition effect of dry cladode of $O$. ficus-indica extracts were from ethanol, petroleum ether, chloroform, and 1butanol extracts (Fig. 4). It is clear that the effectiveness of plant extract is widely affected by the type of solvent. In this study, both organic solvents (polar and non-polar) stimulate precipitation of active chemical compounds with varying relative effectiveness. They showed a moderate-to-slight activity against pathogens that varies from one sample of $O$. ficus-indica to another. Related results demonstrate that the organic extract has significantly varying polarity impact on tested microbes (Sen and Batra 2012).

The results of the antibacterial screening illustrated that all solvents of dry cladode extracts have inhibitory effects against $K$. pneumoniae and all solvents of fresh extracts have inhibitory effects against $C$. albicans. Petroleum-ether dry extracts exhibited a higher degree of antimicrobial activity as compared to other solvent extracts $(2.33 \pm 005 \mathrm{~cm})$ followed by the methanol dry plant extract $(1.76 \pm 0.11 \mathrm{~cm})$. In contrast, the 1-butanol dry extract activity recorded the lowest degree of such activity $(1.10 \pm 0.1 \mathrm{~cm})$ (Fig. 4). Additionally, chloroform and methanol fresh extracts of $O$. ficus-indica cladode displayed promising activity against $K$. pneumoniae (Fig. 5). The extracts of 

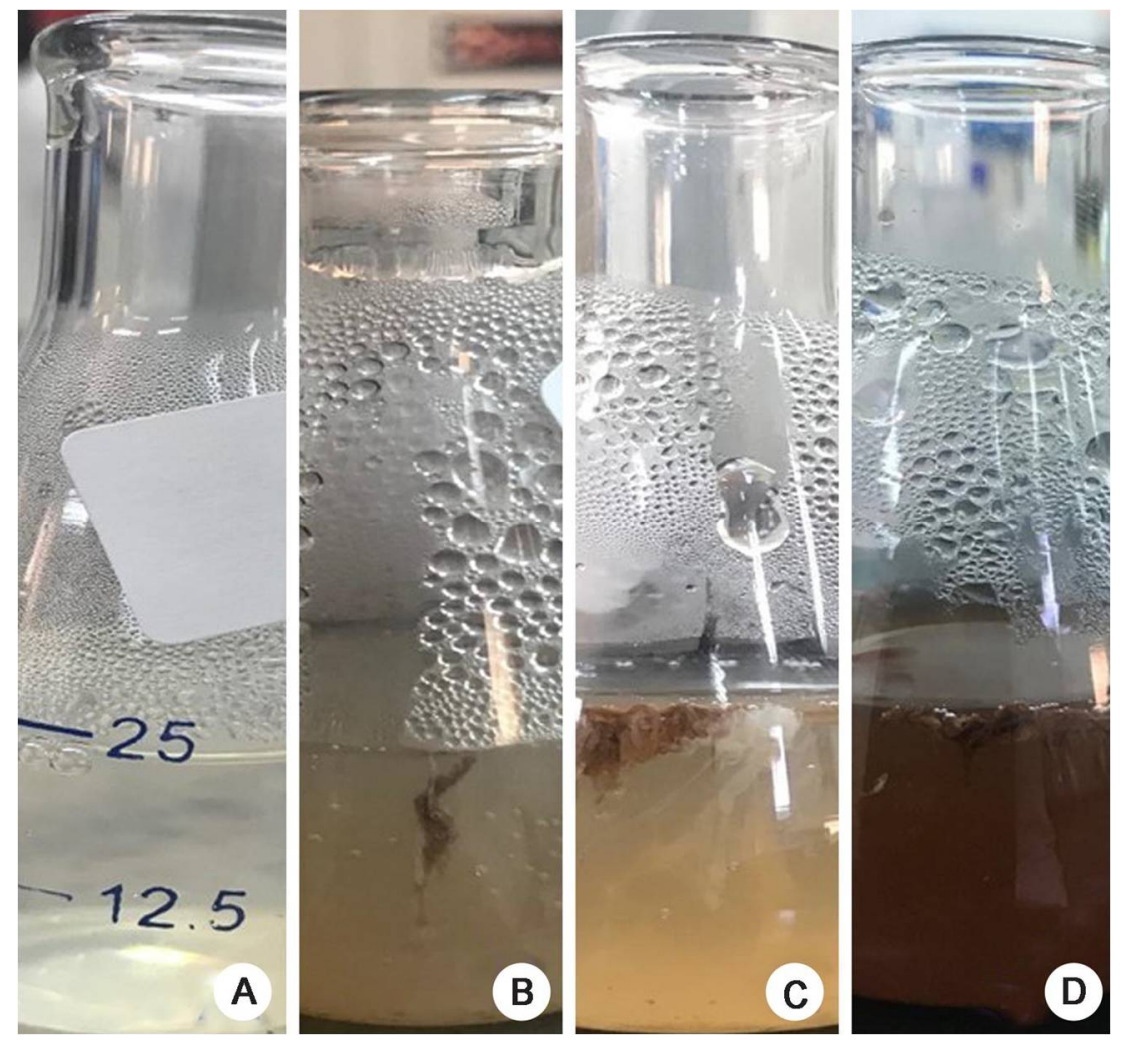

Fig. 1. Synthesis of silver nanoparticles (A) $\mathrm{AgNO}_{3}$ solution, (B) after 10 min synthesized Ag-NPs in yellowcolor solution, (C) after $30 \mathrm{~min}$ turned to brown color solution and (D) after $1 \mathrm{hr}$ formation and stabilization of Ag-NPs in brownish red solution.

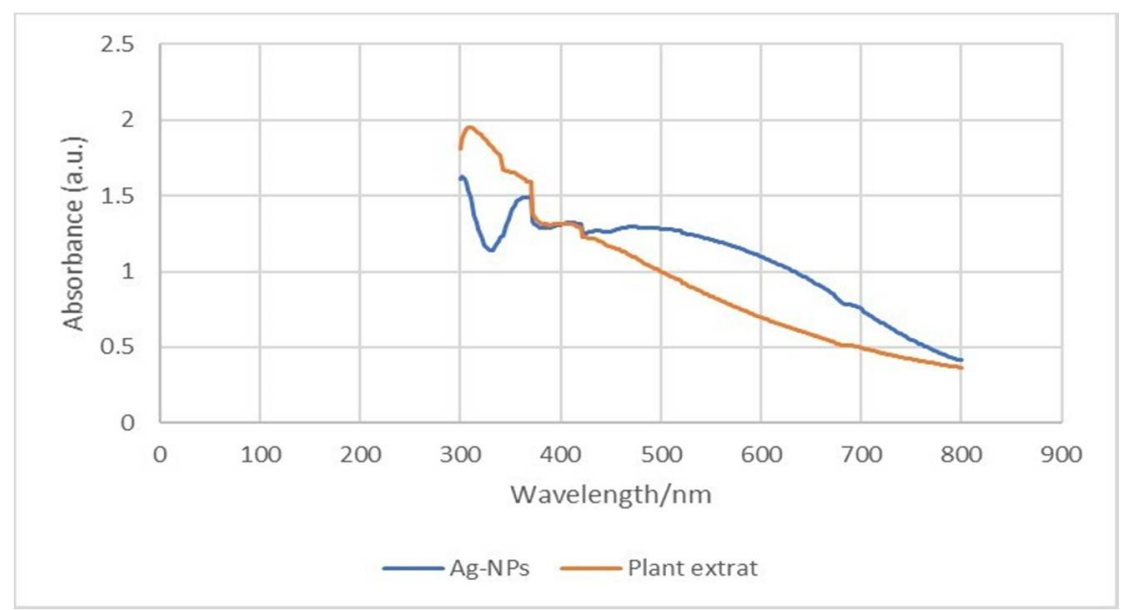

Fig. 2. UV-visible spectra of Ag-NPs solutions synthesized from $O$. ficus-indica cladode extract. 

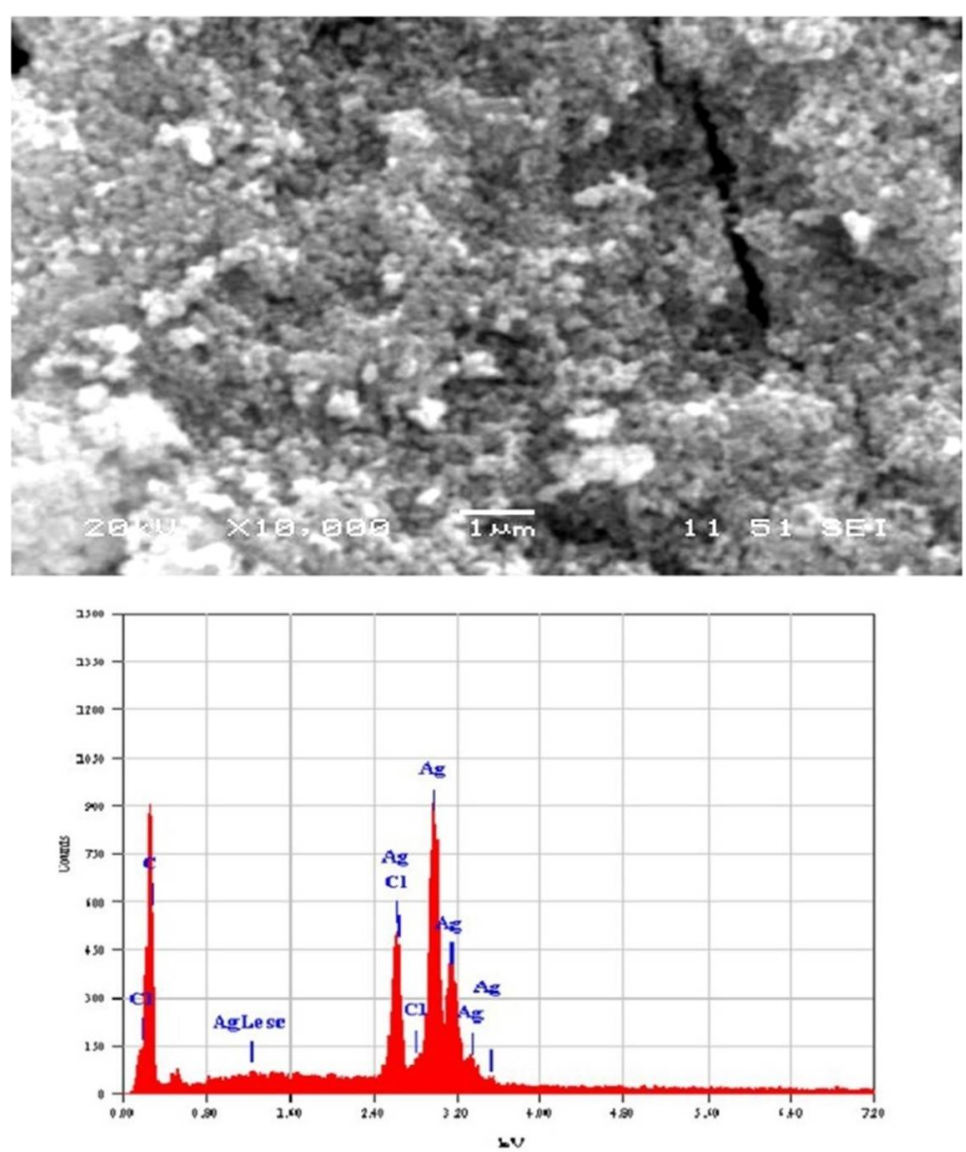

Fig. 3. SEM images of Ag-NP solution synthesized from $O$. ficus-indica cladode and corresponding EDX.

\section{Solvents extract (dry)}

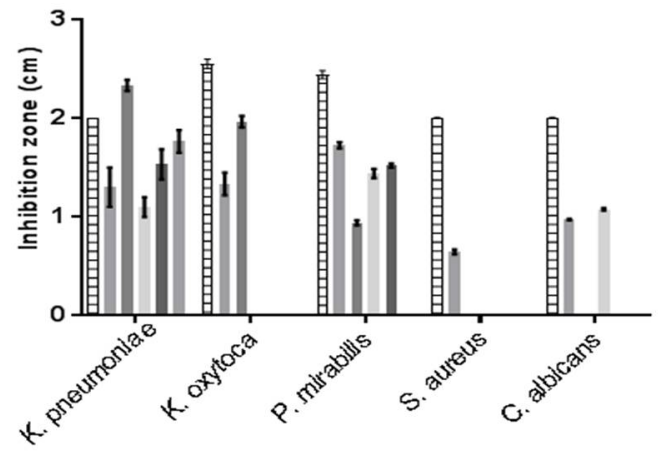
日 P.C.
- Chloroform
- Petroleum ether
-1-Butanol
- Ethanol
- Methanol

Fig. 4. Antimicrobial activity of $O$. ficus-indica dry cladode extracts against pathogenic strains. 


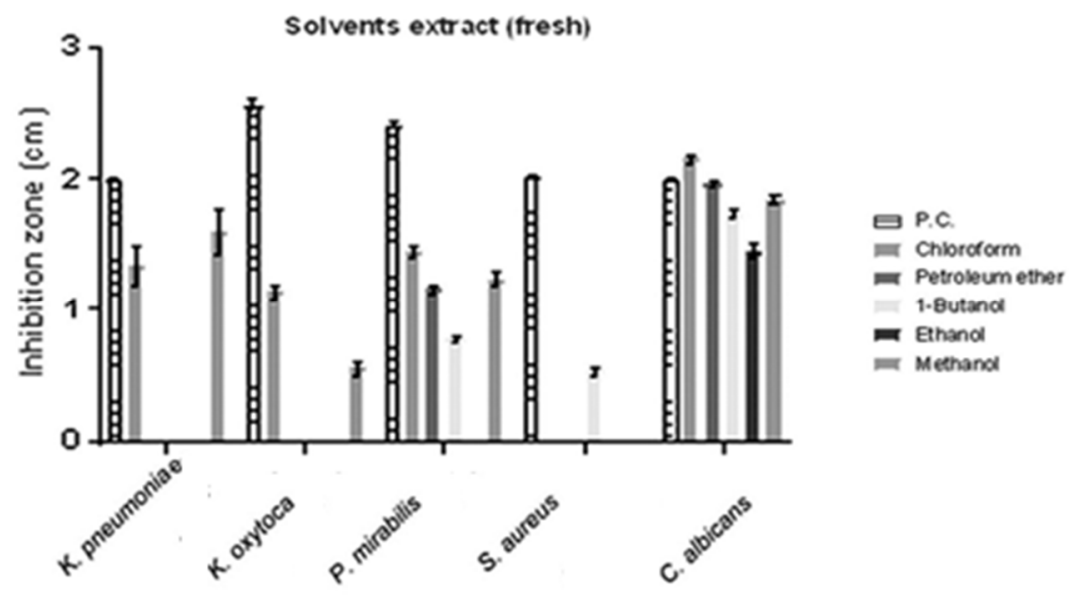

Fig. 5. Antimicrobial activity of fresh cladode extracts of $O$. ficus-indica against pathogenic strains.

the dry and fresh plants tested were found to be active against $K$. oxytoca. Fig. 4 clearly illustrated that the solvent dry extracts from cladode of $O$. ficus-indica had a narrow antibacterial activity. The highest inhibitory activity was recorded from the petroleum ether and chloroform dry extracts $(1.96 \pm 0.05$ and $1.33 \pm 0.11 \mathrm{~cm})$. Fig. 5 illustrates the promising inhibition activity against $K$. oxytoca, which was gained only from chloroform, and methanol fresh extracts of $O$. ficus-indica cladode $(1.13 \pm 0.05$ and $0.55 \pm 0.04 \mathrm{~cm})$. In case of antibacterial activity against $P$. mirabilis, the highest antibacterial potential observed from plant dry extract are as follows: chloroform dry extract represented the highest activity $(1.76 \pm 0.03 \mathrm{~cm})$, followed by ethanol and 1-butanol extracted $(1.52 \pm 0.02$ and $1.44 \pm 0.04 \mathrm{~cm})$. However, petroleum ether dry extract exhibited less antibacterial activity $(0.94 \pm 0.02 \mathrm{~cm})$. The results also demonstrated that solvents extracted from the fresh cladode of $O$. ficus-indica had less activity than dry extract and the positive control, which had a narrow spectrum against $P$. mirabilis. Chloroform fresh extract exhibited the maximum activity $(1.44 \pm 0.04 \mathrm{~cm})$, followed by methanol $(1.24 \pm 0.04 \mathrm{~cm})$, while 1-butanol extract showed the minimum value of inhibition zone $(0.76 \pm 0.02 \mathrm{~cm})$. 1-butanol and chloroform extracts from the dry cladode showed potential of anticandidal activity against pathogenic $C$. albicans $(1.07 \pm$ 0.01 and $0.97 \pm 0.01 \mathrm{~cm}$ ). On the other hand, all extracts from $O$. ficus-indica fresh cladode have inhibitory effects against the tested Candida. The strongest spectrum of activity attained from the chloroform extract $(2.15 \pm 0.03 \mathrm{~cm}$ ), which was greater than the positive control (Cefoxitin dissolved in DMSO) and other solvents. However, ethanol extract recorded the minimum activity $(1.45 \pm 0.04 \mathrm{~cm})$.

This difference in activity between different organic extracts may be due to the variations between extract compounds in the fresh and dry cladode of $O$. ficus-indica extract. This finding is in general agreement with the result of Cowan (1999). Preethi (2010) mentioned that the alcoholic extract had a high degree of antimicrobial activity in different medicinal plants while petroleum ether extract exhibits intermediate and aqueous extract showed a low degree of antimicrobial activity. In addition, no stable chemical compound or volatile oil may be changed through drying process, such as the fresh samples containing the mucilage layer that coating polysaccharides with high molecular weights that are difficult to dissolve in water or may be in other solvents. Furthermore, perhaps the time of collection and storage conditions could have contributed to the 
contrasting results (Olafimihan 2004). Existence of polyphenols (limited solubility in water), calcium oxalate, and compounds of flavonoids was found to have antimicrobial activity (El-Feghali et al. 2018).

Fig. 6 showed antimicrobial activity with Ag-NP solutions against pathogenic bacteria and pathogenic candida. The antimicrobial activity of Ag-NP solutions against $K$. pneumoniae and $C$. albicans comprised of the widest spectrum of all pathogens $(2.03 \pm 0.05 \mathrm{~cm})$. Contrarily, $P$. mirabilis was the lowest value $(1.82 \pm 0.02 \mathrm{~cm}$ ), compared to the positive control (Cefoxitin dissolved in DMSO). In this work, slight susceptibility differences between pathogenic microorganisms were observed along with a significant inhibitory effect of Ag-NP solutions. The findings of this study are not in agreement with the results obtained by Metwally et al. (2018),

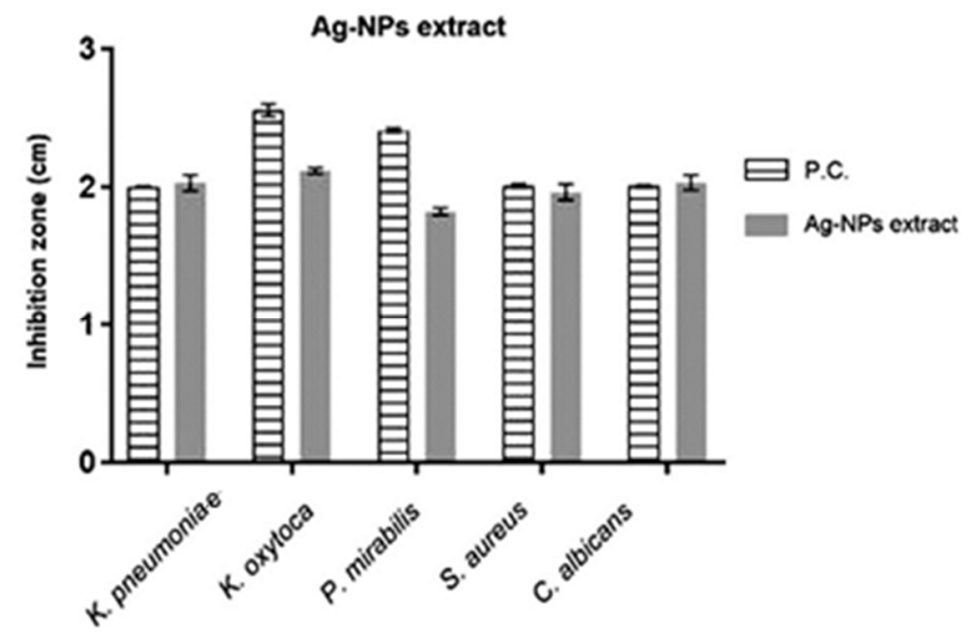

Fig. 6. Antimicrobial activity of silver nanoparticles synthesized from $O$. ficus-indica cladodesextracts against pathogenic microorganisms.

who reported that Gram-negative bacteria was less affected by silver-NP extracts than Grampositive bacteria due to the positive charge of silver NPs that interact with the Gram-negative lipopolysaccharide. This result again supports that in the extract of $O$. ficus-indica plants, there were specific chemical compounds targeted to the positive bacterial strains. Petica et al. (2008) have reported that silver nanoparticles exert the same effect on Gram-positive and Gram-negative bacteria and a fungal mix of Aspergillus, Penicillium and Trichoderma species. This study also reported that Gram-positive bacteria (S. aureus) are more resistant to plant extracts than Gramnegative bacteria ( $P$. mirabilis, Klebsiella spp.), which were inhibited by most $O$. ficus-indica dry and fresh cladode extracts. The Gram-negative hydrophilic surface of the bacterial cell wall is thought to act as a barrier to many unusual substances, including antibiotics (Nikaido 1994). It is speculated that particular chemicals may be working specifically for Gram-positive rather than negative bacteria.

These results indicated that there is a considerable difference in antimicrobial activity between solvent extracts and Ag-NP solutions. The Ag-NP solution showed that there is hyper-inhibitory activity against tested microbes that differs from extract of cladode for all solvents whereas extracts showed a high degree of specific antimicrobial activity against $C$. albicans and $K$. pneumoniae. The difference is due to the nanoparticles having large surface areas, which allow them to penetrate the pathogenic cell wall more easily by attaching Ag-NPs to its cell membrane, 
interact with it, then enter and damage the cell. This mechanism indicates the efficiency of Ag-NPs antimicrobial activity properties as confirmed by other researchers (Morones et al. 2005).

These solvent extracts and synthesized AgNPs obtained from $O$. ficus-indica cladodes which proved to be potentially effective against human pathogenic microbes could be used as natural alternative drugs for microbial control than synthetic chemicals.

\section{References}

Abouhosseini M, Youssefi M, Ghasemi F, Ghias TR, Haji ER and Yousefi BM 2012. Comparison of antibacterial effects of Eucalyptus essence, Mint essence and combination of them on Staphylococcus aureus and Escherichia coli isolates. World Appl. Sci. J. 16(10): 1473-1477.

Ahmed S, Ahmad M, Swami BL and Ikram S 2016. A review on plants extract mediated synthesis of silver nanoparticles for antimicrobial applications: A green expertise. J. Adv. Res. 7(1): 17-28.

Cowan M 1999. Plant products as antimicrobial agents. Clin. Microbiol. Rev. 12: 564-582.

Dastagir G, Hussain F and Khan A 2012. Antibacterial activity of some selected plants of family Zygophyllaceae and Euphorbiaceae. J. Med. Plant Res. 6(40): 5360-5368.

El-Feghali PAR, Ibrahim R and Nawas T 2018. Antibacterial activity of Curcuma longa, Opuntia ficusindica and Linum usitatissimum. MOJ Toxicol. 4(3): 214-220.

Ennouri M, Ammar I, Khemakhem B and Attia H 2014. Chemical composition and antibacterial activity of Opuntia ficus-indica F. Inermis (Cactus Pear) Flowers. J. Med. Food 17(8): 908-914.

Galati EM, Monforte MT, Miceli N, Mondello MR, Taviano MF, Galluzzo M and Tripodo MM 2007. Opuntia ficus indica (L.) Mill. mucilages show cytoprotective effect on gastric mucosa in rat. Phytother. Res. 21(4): 344-346.

Lee EH, Kim HJ, Song YS, Jin C, Lee KT, Cho, J, and Lee YS 2003. Constituents of the stems and fruits of Opuntia ficus-indica var. saboten. Arch. Pharmacal. Res. 26(12): 1018-1023.

Martin I, Sawatzky P, Liu G and Mulvey MR 2015. STIs and sexual health awareness month: Antimicrobial resistance to Neisseria gonorrhoeae in Canada: 2009-2013. Can. Commun. Dis. Rep. 41(2): 35-41.

Matu EN and Van J 2003. Antibacterial and anti-inflammatory activities of some plants used for medicinal purposes in Kenya. J. Ethnopharmacol. 87(1): 35-41.

Morones JR, Elechiguerra JL, Camacho A, Holt K, Kouri JB, Ramfrez JT and Yacaman MJ 2005. The bactericidal effect of silver nanoparticles. Nanatechnol. 16: 2346-2353.

Moustafa MF, Alamri S, Taha TH and Alrumman SA 2013. In vitro antifungal activity of Argemone ochroleuca Sweet latex against some pathogenic fungi. Afr. J. Biotechnol. 12(10): 1132-1137.

Nikaido H 1994. Porins and specific diffusion channels in bacterial outer membranes. J. Biol. Chem. 269(6): 3905-3908.

Olafimihan CA, 2004. Effects of seasonal variation on the antibacterial activity of aqueous extract of Azadirachta indica fresh stem bark. Biosci. Res. Commun. 16(1): 13-16.

Parekh J and Chanda S 2007. In vitro antimicrobial activity of Trapa natans L. fruit rind extracted in different solvents. Afr. J. Biotechnol. 6(6): 766-770.

Petica A, Gavriliu S, Lungu M, Buruntea N and Panzaru C 2008. Colloidal silver solutions with antimicrobial properties. Mater. Sci. Eng. 152: 22-27.

Prabhu S and Poulose EK 2012. Silver nanoparticles: Mechanism of antimicrobial action, synthesis, medical applications, and toxicity effects. Int. Nano. Lett. 2(1): 32, 1-10.

Preethi R, Devanathan VV and Loganathan M 2010. Antimicrobial and antioxidant efficacy of some medicinal plants against food borne pathogens. Adv. Biomed. Res. 4(2): 122-125.

Salvat A, Antonacci L, Fortunato RH, Suárez EY and Godoy HM 2004. Antimicrobial activity in methanolic extracts of several plant species from Northern Argentina. Phytomedicine 11(2): 230-234.

Sen A and Batra A 2012. Evaluation of antimicrobial activity of different solvent extracts of medicinal plant: Melia azedarach L. Int. J. Curr. Pharm. Res. 4(2): 67-73. 
Song JY and Kim BS 2009. Rapid biological synthesis of silver nanoparticles using plant leaf extracts. Bioprocess Biosyst. Eng. 32(1): 79-84.

Trombetta D, Puglia C, Perri D, Licata A, Pergolizzi S, Lauriano ER and Bonina FP 2006. Effect of polysaccharides from Opuntia ficus-indica L. cladodes on the healing of dermal wounds in the rat. Phytomedicine 13(5): 352-358.

(Manuscript received on 23 December, 2019; revised on 16 April, 2020) 